\title{
Thirty-Fifth Annual AOA Research Conference Abstracts, 1991: Part 1
}

Part 1 contains abstracts of papers to be presented at the ThirtyFifth Annual AOA Research Conference in the Burroughs Wellcome Fellows, Biomedical Sciences, and Clinical Sciences categories. For the convenience of attendees, abstracts appear in their scheduled sequence, and are numbered for easy reference. Part 2, Abstracts of presentations in the Poster Sessions, will appear in the October issue.

Abstracts are reproduced exactly as submitted. The American Osteopathic Association and The Journal of the American Osteopathic Association assume no responsibility for the content of the abstracts printed herein.

\section{Burroughs Wellcome Fellows F08}

A KINEMATIC MODEL OF COMMON COMPENSATORY PATTERN.

R.H. Johnson, B.S. and N.A. Cross, PhD.,

UNECOM OPP/Anatomy Depts, Biddeford, Me. 04005 .

Common compensatory pattern (CCP) in the lumbar spine has been quantified. The incidence of CCP in an asymptomatic population has been established. The lumbar spine that is in CCP has the left superior facet oriented more frontally. These asymmetries predispose the lumbar spine to imbalance and instability that may manifest in vertebral rotation

These asymmetries are created through a combination of genetic, developmental and behavioral influences. The superior articular facets are oriented in the frontal plane superior articular facets are oriented in the frontal plane prenatally and gradually rotate lnto the sagittal plane. There is an asym. rly childhood.

Postural asymmetries of CCP will place unequal loading on the facets. It has been estimated that the facets carry up to 308 of the load, increasing to 478 in degenerative disc disease. The forces of preload, physiologic loads and other forces are sufficient enough to caure a remodelling of the facet bony architecture, thereby leaving a measureable asymmetry.

Models of spinal motion, kinematics and biomechanics assume that there is some theoretical normal. Yet, there is a large degree of scatter noted in most motion studies of the spine. spine. These studies assume bilateral symmetry, which is clearly not present. The left facet is more frontally any models of spinal motion have to take into consideration these asymmetries. The theoretical normal does not exist!

When a compressive force is applied to a lumbar motion gegment with the left facet frontally oriented, there is resultant right rotation of the vertebral body. Sidebending to the left will be greater than to the right in this situation. The facets may influence the motion of the lumbar vertebra to a greater extent in extension than in flexion. vertebra to a The person that exhibits CCP will be using greater muscular effort to maintain posture and for loc

These asymmetries also predispose the lumbar spine to pathologies, such as osteoarthritis, disc disease, facet ayndromes and low back pain.

\section{F03}

THE EPIDEMIOLOGY OF ANDROGENETIC ALOPECIA

ML LEAVITT DO; JW YOUNG DO; AL SCHROETER MD; R MARKERT PHD; TY CHUANG MD; SA FRIEDMAN DO; ET CONTE DO

Grandview Hospital Medical Center, Department of Dermatology Dayton, Ohio 45405

Androgenetic alopecia (AGA) affects a great portion of the population of the U.S. It has always been presumed to result largely from heredity and its epidemiology has, to our knowledge, never been studied. We sought to define more about this entity, thinking if more was known about it's cause, more could be done in it's treatment.

A questionnaire was developed, asking a broad variety of questions relating to an individual's age, heredity, health, sun exposure, occupation and skin. 455 questionnaires were returned. We used chi-square analysis with Yates correction for continuity to interpet the information.

$359(80 \%)$ were males and $91(20 \%)$ were female. $391(86.3 \%$ were white and $35(7.7 \%)$ were black. The mediage age was 35.5 years. Respondants were grouped into less hair loss (men=Hamilton 1,2 3; women=Ludwigs 0,1 ) and more hair loss (men=Hamilton 4 or greater; women-Ludwigs 2 or 3 ).

People with more hair loss tended to be older (approaches statistical significance, $p=0.051$ ) and have at least 1 first degree relative with hair loss $(p=0.04)$. Personal sun exposure history, skin type and eye color do not seem to be associated. Interestingly, more frequent (once daily or more) shampooing is associated with more hair loss $(p=0.04)$ as is a history of wearing a helmet or hat that caused scalp sweating $(p=0.05)$. Not surprisingly, people with more hair loss tend to wear hats more often $(p=0.004)$ and be less happy with their physical appearance $(\mathrm{p}=0.04)$.

There appears to be no correlation with general health, skin disease, smoking thyroid disease, anemia, medications, or autoimmune disease. People with more hair loss tend to have more body hair $(p=0.0001)$.

Supported by Burroughs-We11come Grant F-90-03. 


\section{F06}

Determination of Niacin Nutriture: Implications for Prevention of Carcinogenesis. Bryan L. Wasson, Elaine L. Jacobson, Hai-Ying Chen, Jia-Jen Chen, and Myron K. Jacobson. Texas College of Osteopathic Medicine, University of North Texas, Fort Worth, Tx 76107 USA

The amount of dietary niacin necessary to prevent pellagra is well known. However, a growing body of evidence suggests a new role for the active form of this vitamin, NAD. It is now known that the conversion of NAD to polymers of ADP-ribose is required for repair of carcinogen induced DNA damage. The enzyme that catalyzes this reaction requires intracellular NAD concentrations in the range of 100-300 $\mu \mathrm{M}$. This requirement raised the question of whether current human niacin nutriture is sufficient to provide optimal tissue NAD levels for protection against carcinogenesis.

We initiated the development of a rapid, sensitive method for determination of niacin status that could be utilized for large population studies. Recent studies have shown that the ratio of NAD to NADP in human erythrocytes reflect niacin nutriture (Fu, et al., J. Nutr., 119: 1949-1955, 1989). We have studied the distribution of NAD in fractionated blood components and shown that $98 \%$ of the NAD and NADP in whole blood is derived from erythrocytes. Therefore, a method to extract whole blood that was suitable for NAD and NADP determinations was developed. The following criteria were proposed and met: (1) The amount of NAD and NADP measured must be proportional to the amount of extract assayed. (2) The NAD and NADP in the extract must be stable. (3) The extract components must not interfere with the assay. (4) The method must be reproducible. (5) The NAD and NADP in blood samples must remain stable during storage and transport to the laboratory. We have referred to the ratio of NAD to (NAD + NADP) $x$ $100 \%$ as niacin number. This number was found to be $64 \pm 4$ in adult males consuming the RDA for niacin. Assessment of the range of niacin number in a control population and the relationship of dietary niacin to niacin number is in progress in a prospective case cohort study of diet and cancer. Supported in part by a Burroughs Wellcome AOA Fellowship to BLW and NIH grant CA43894.

\section{F05}

CLINICAL RESULTS OF BIPOLAR HIP ARTHROPIASTY

D. W. Prieskorn, D.O.; P. Burton, D.O, ; J Swienckowski, D.O.; B. J. Page, D.O.: Botsford General Hospital; Department of orthopaedic Surgery; Farmington Hills, Michigan 48335

(Abstract not available for publication)

\section{F04}

\section{Osteopathic Diagnostics in Breast Cancer Therapeutics}

Thomas E. Merchant, DO and Anthony Dekker, DO

Division of Community Medicine, Chicago College of Osteopathic Medicine, 5200 South Ellis Avenue, Chicago, Illinois 60615 USA

Osteopathic structural examinations were performed on 64 female patients prior to breast cancer surgery and 7,21 and 90 days post-operatively. An axillary dissection was performed in all cases. Lymphedema, extremity range-of-motion, spinal curves, paraspinal and extremity soft-tissue changes and the levels of bony landmarks were assessed. The most consistent findings were noted in range of abduction and soft-tissue changes of the ipsilateral arm and in the assessment of the acromioclavicular (AC) levels.

A tilt in the AC levels toward the side of the affected breast was noted preoperatively in $92 \%$ of the examined patients. At 3 months, a compensatory tilt in the $\mathrm{AC}$ level away from the affected side was noted in $80 \%$ of the patients. Except for patients experiencing complications, abduction of the ipsilateral arm was found to be time dependent: $45^{\circ}$ at 7 days, $90^{\circ}$ at 21 days and $180^{\circ}$ at 90 days.

Through a better understanding of somatic changes as a result of breast cancer therapy, measures could be taken to reduce the debilitating consequences of surgery and the overall time for recovery. Somatic complaints, deformity and loss of function, once an accepted consequence of breast cancer therapy, may be reduced through osteopathic diagnostics and therapeutics.

\section{F02}

FUNCTION DURING CONVENTIONAL TREATMENT OF

DYSPHAGIA J. M. Krettek, D.O., Chicago College of Osteopathic Medicine, Center for osteopathic Research and Educational Development, Chicago, Illinois 60615

This study, approved by the IRB, observes the course of somatic dysfunction during a three month period of conventional treatment of dysphagia. In order to study the effects of osteopathic Manipulative Treatment (OMT) on these somatic dysfunctions and dysphagia, one needs to know their general course with current forms of treatment.

Patients were selected from those who were referred to the Olympia Fields Osteopathic Medical Center's radiology department for videofluoroscopic examination. Those included were 1) patients over 18 years of age, 2) patients who experienced dysphagia within the past month, 3) patients who were able to communicate and cooperate with the examination process. Patients were interviewed and examined by a speech pathologist and underwent a videoesophagram. The researcher's osteopathic exam was conducted without access to the previously mentioned data until the research was completed. Patients were interviewed and examined at three separate visits over a 6 month period of time.

This is a preliminary study. No statistical analysis was run on this small sample. The results of the study are to be compared with published information in this field. There are four main diagnoses for the seven patients in this study. The osteopathic findings are described in terms of their location, intensity, character, and persistence. Four diagnoses were shared by the seven patients, chronic pharyn-gitis and tonsillar hyperplasia, seizure disorder, gastroesophageăl reflux, and stroke/transient ischemic attacks affecting four of the seven. The last four cases were associated with upper cervical and upper thoracic findings. The course of these lesions was compared with the subjective reports of patients' symptoms. 


\section{Biomedical Sciences}

\section{1}

RESTRAINT STRESS INCREASES INDUCED PERSISTING HINDLIMB FLEXION (SPINAL FIXATION) IN RATS. M.M. Patterson. Ph.D. M.J. Bartelt M.S. E.J Jehnson. B.S. and A. Howe. B.S. Department of Psychology and College of Osteopathic Medicine, Ohio University. Athens Ohio, 45701

Lasting hindlimb flexion (spinal fixation) may be induced by external stimulation to the hindlimb in spinalized rats (Steinmetz et. al., J.C.P.P., 95:4, 548-555, 1981).Based on the work of Bartelt and Patterson (Neurosci. Abst.,15:467, 1989; Neurosci. Abst. 16:1238 1990) which suggested that a number of factors may increase fixation, the present study investigated the contribution of stress to the fixation. Thirty-six rats were randomly assigned to a control (no stress) group and a restraint stress group. In the stress group the animals were placed in a restraint box for thirty minutes. Immediately following the stress (or no stress/control) the animals were anesthetized (Nembutal, $50 \mathrm{mg} / \mathrm{kg}$ i.p.), a spinal transection performed and the upper hindlimb stimulated with 3-4mA,100-pps, $7 \mathrm{msec}$, repetitive $\mathrm{dc}$ pulses for $40 \mathrm{~min}$. The persisting hindlimb flexion was measured as the amount of weight needed to remove the asymmetry following stimulation. The experimenter was unaware of the animal's assignment. The results demonstrate a significant increase in postural asymmetry $(p<.05)$ induced by the restraint stress condition.

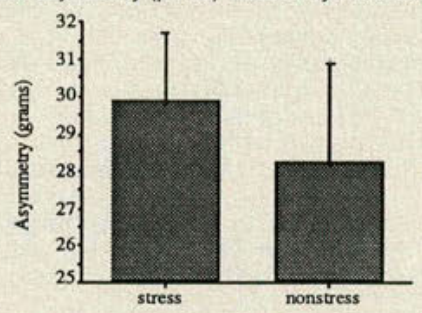

This finding is striking because the induced stress is mild and yet increases induced hindlimb flexion. This suggests that a generalized organismic stress may enhance induced alterations of spinal reflex excitability. It may be that long-term alterations in spinal excitability (which may occur frequently as a result of chronic pain-producing somatic and visceral malfunctions) are enhanced by environmental or physical stress, producing long-term somatic dysfunction.

Support: American Osteopathic Assoc. Bureau of Research Grant 90-08-319.

\section{2}

DNA HOMOLOGIES OF PUTATIVE ACINETOBACTER SP. CLINICAL ISOLATES AND THE APPLICATION OF DNA HYBRIDIZATION FOR POSITIVE IDENTIFICATION. J.B. oliver, B.S., J.D. Jollick, Ph.D. Ohio University College of Osteopathic Medicine. Athens, ohio 45701

Members of the genus Acinetobacter are among the glucose nonfermenting, gram negative bacteria. As such they do not possess any unique phenotypic characteristics that make possible ready identification and differentiation of these bacteria from other similar organisms. However, DNA homology in Acinetobacter is a useful discriminating test. A rapid DNA hybridization test for identification of Acinetobacter, which is the goal of this study, would provide a valuable tool for research and eventually diagnostic medicine.

Genomic DNA from Acinetobacter calcoaceticus var. anitratum, $\mathbb{A} . \underline{\mathrm{C}}$. var. 1 woff $i$, and $\mathbb{A}$. haemolyticus was isolated, and purified. ${ }^{-}$cloned DNA fragments were used to probe for homologous DNA sequences in target organisms. The target organisms were prepared from clinical bacterial isolates (obtained from hospitals in the Eastern U.S.) putatively identified as A. C. vars. anitratum, lwoffi or just A. calicoaceticus. The isolates were originally found in peritoneal dialysis fluid, blood, cerebral spinal fluid, heart blood (autopsy), wound cultures etc. The clinical strains were grouped by DNA homology.

We found four distinct groups among clinically important strains of Acinetobacter. DNA hybridization was found to be an accurate method for differentiating acinetobacters from similar control organisms. The methods used in this study could be modified for the rapid identification of acinetobacters in a clinical setting.

\section{3}

MALIGNANT FIBROUS HISTIOCYTOMA (MFH): PRESENCE OF KBRATIN FILAMENTS AS VISUALIZED WITH IMMUNOSTAINING AND ELECTRON MICROSCOPY. K.T. Fisher, B.S., Wm.D. Meek, Ph.D., College of Osteopathic Medicine-0klahoma State University, Department of Anatomy, Tulsa, Oklahoma 74107.

A unique case of MFH exhibited cells containing keratin intermediate filaments (IFS) and other morphological distinctive features that have been reported rarely in the literature. A supraorbital mass was removed from a $78 \mathrm{yr}$. old male patient and sent to the Electron Microscopy Diagnostic Center at COM-0SU. The specimen was further prepared for transmission electron microscopy (TBM) and immunolabelling. An ABC immunoperoxidase technique was used with antibodies to the IF proteins desmin, keratin, and vimentin. Light microscopy revealed a poorly differentiated spindle cell tumor of the dermis and subcutaneous tissues with significant pleomorphism and a striking storiform pattern. Occasional giant cells were present. The tumor cells were positive for both vimentin and keratin but negative for desmin. Internal controls of skeletal muscle showed a positive reaction for desmin and the stratified squamous epithelium showed positive for keratin. TEM revealed spindle-shaped to rounded fibroblast-like tumor cells in a collagenous stroma. Myofibroblasts vere present with long actin bundles and related actinin densities. Many of these bundles vere closely applied to the cell membrane and interacted with fibronectin forming a fibronexus. Bistiocytes containing prominent Golgi and numerous lysosomes vere seen, in addition to, primitive mesenchymal cells. Keratin IFs were arranged in the typical swirled, bundled fashion. This case exhibited the classic features of MFH but also contained cells vith keratin IFs. These results show the diversity of MFH origin and/or the ability of tumor cells to coexpress intermediate filament proteins. Supported in part by Grant No. 89-09-245 from the American Osteopathic Association.

\section{4}

RELATIONSHIP BETUEEN MICROFILAMENTS, INTBRMEDIATE

FILAMENTS, AND MICROTUBULES IN MITOTIC HUMAN AMNION YISH CELLS. C.J. Pitcock, B.S., Ym.D. Meek, Ph.D., College of 0steopathic Medicine-0klahoma State University, Department of Anatomy, Tulsa, Oklahoma 74107. The function of intermediate filaments (IFs) in mitosis is a subject of interest as more becomes known about the biochemistry, physiology, and morphology of the cytoskeletal network. This study deals vith the positional or morphological relationship of IFs with other members of the cytoskeleton during mitosis of a transformed human cell line. Colabelling of the cytoskeleton in various combinations: microtubules (Mts) with fluorescein isothiocyanate (FITC) and microfilaments (Mfs) with rhodamine phalloidin (RD-PD), IFs with FITC and Mfs with RD-PD, allowed visualization in the same cell. The various stages of cells in mitosis were photographed, being quickly identified by either the mitotic spindle or round shape. Cells were also prepared for transmission electron microscopy in order to study the relationship of microfilaments with keratin densities and the IFs. Cytochalasin D treatment was used to test the relationship of $\mathrm{Mfs}$ and IFs. Short but intact keratin IFs vere present in some mitotic cells but other cells shoved the characteristic dense, spherical keratin bodies (Meek, et al, 1990). Vimentin IFs vere longer and more evident in mitotic cells, sometimes clustered around the chromosomes. Actin occupied its typical position in these cells: beneath the cell membrane, vithin the cleavage furrow, and within microvilli. Vimentin appears to have more of a role than keratin in WISH mitotic cells, although the keratin densities are not present in every mitotic cell and may serve to interact with other cells in junctional complexes or interact with keratin IPS in stabilizing the movement of chromosomes. 
THE USE OF NEURAL NETWORKS TO ASSESS THE DIFFERENTIAL DIAGNOSTIC SKILLS OF MEDICAL STUDENTS R. C. Stone, B.S. \& F.J. Papa. D.O. Ph.D.

Texas College of Osteopathic Medicine, Department of Medical Education

Fort Worth, Texas, 76107

Medical educators suggest that there are significant psychometric limitations to the assessment instruments used to measure the differential diagnosis (DDX) skills of medical students. The department of Medical Education at TCOM has been aggressively pursuing the use of artificial intelligence based tools as an alternative assessment methodology. The objective of this study was to perform a preliminary investigation of the psychometric properties (reliability and validity) of a neural network based DDX assessment instrument.

The Parallel Distributed Processing Back Propagation neural network was selected to function as the DDX assessment instrument. The DDX skill area chosen for study involved the chief complaint of "Weakness". A weakness knowledge base consisting of the relationships between four common causes of weakness and 18 associated signs and symptoms was extracted from 64 third and fourth year medical students. Each studentderrived knowledge base was used by the neural network to diagnose 97 actual weakness cases.

The diagnostic behavior (accuracy) achieved by the neural network operating with the student-derrived knowledge base was not random $\left(X^{2}, \mathrm{p}<.001\right)$.Test reliability was very high (Kuder-Richardson-21 $=.92$ ).

Additional studies are necessary to substantiate the construct validity of this DDX assessment instrument. Traditional DDX assessment instruments rarely achieve reliability coefficients greater than .80 . These preliminary findings suggest a clear "reliability" advantage provided by a neural network DDX assessment instrument.

This study was in part funded by AACOM FOCUS/SmithKline Beecham.
PURIFICATION OF CHOLESTEROL SULFOTRANSFERASE FROM RAT

SKIN. J.I.Rearick, Ph.D. Kirksville College of Osteopathic Medicine, Department of Biochemistry, Kirksville, MO 63501.

The objective of this study is to develop methods to purify the enzyme, cholesterol sulfotransferase (CST). Pure CST is required to generate molecular probes (such as antiCST antibodies and CST CDNA) which will be useful in determining how CST activity is regulated (transcriptionally, translationally or posttranslationally). Airway epithelial cells or epidermal keratinocytes dramatically up-regulate CST activity during squamous differentiation. This increase is prevented by retinoids.

Neonatal rat skin was chosen as an enzyme source. An assay was developed using adenosine $3^{\prime}$-phosphate, $5^{\prime}$-phospho[35S]-sulfate (PAPS) as the sulfate donor and cholesterol as the sulfate acceptor. Cholesterol [ 35 S]-sulfate was separated from PAPS by organic solvent extraction and partitioning against an aqueous phase. High-speed supernatants of skin homogenates contained all the CST activity, suggesting that CST is a cytosolic enzyme. Chromatography on DEAE-Sephacel led to a 15 -fold purification in quantitative yield. Chromatofocussing suggests CST has a pI near pH 4.2.

Kinetic analyses of the partially purified CST yield a $K_{M}$ (PAPS) $=1 \mu \mathrm{M}$ and $K_{1}=1 \mu \mathrm{M}$ for adenosine $3^{\prime}, 5^{\prime}$ bisphosphate (PAP), suggesting that PAP-agarose may serve as an effective affinity adsorbent for CST.

Supported by the American Cancer Society and the Charles and Jennie Fermaturo Charitable Foundation.

\section{6}

THE EFFECT OF DANAZOL ON TISSUE PLASMINOGEN ACTIVATOR IN THE RAT. A. K. Will ingham, Ph.D., R. L. Bartels, B.A., V. A. Schekorra, 0.0 . and J. E. Malick, 0.0 ., Departments of Biochemistry and Obstetrics/Gynecology, Kirksville College of Osteopathic Medicine Kirksville, MO 63501. Endometriosis, the presence of functioning endometrial glands and stroma outside the uterine cavity, is a significant problem in $10 \%$ of all reproductive aged women, causing infertility and pelvic pain. It has been suggested that these extra uterine endometrial implants result from depression of the peritoneal fibinolytic system. However, to date no attempts have been made to directly stimulate this system as a method for treating endometriosis. The present study is designed to determine whether the drug danazol has this ability. Danazol is an impeded androgen with antigonadotrophic effects used in the

treatment of endometriosis. We postulate that in the rat, danazol may directly increase plasminogen activator in peritoneal and/or uterine tissue. Danazol was mixed with ground rat food and fed to female rats. Tissue plasminogen activator (TPA)was assayed in peritoneal and uterine tissues. Both tissues examined responded to druc treatment by increased growth. The specific activity of uterine TPA was significantly higher (about $25 x$ ) than peritoneal tissue and rose by about 1.5 to $2 x$ as a result of danazol treatment. The lower specific activity of TPA observed in peritoneal tissue remained relatively constant after treatment. There was no detectable plasminogen activator inhibitors present in these tissues. (Supported in part by a grant from the American Osteopathic Association and the Warner Fund from the Kirksville College of Osteopathic Medicine).

\section{8}

THE USE OF POLYMYXIN TO DETECT ENDOTOXINS. D.M. Driscoll. M.A. Harvard University and Chicago College of Osteopathic Medicine, Chicago, Mlinois, 60515.

The purpose of this research was to study the binding of polymyxin B sulfate to endotoxins (lipopolysaccharides) as a first step toward developing an assay for the detection of lipopolysaccharides in blood. The question I wished to answer was; "Can polymyxin be used to detect endotoxins?"

In this study I made progress toward developing an assay. Specifically, I tested polymyxin B sulfate for its binding properties in vitro to lipopolysaccharides (LPS). The data I used was collected from gel chromatography, fluorometry and spectrophotometry. LPS Fractions with and without polymyxin were placed over a $20 \mathrm{ml}$ gel filtration column packed with S-300 Sephacryl beads, eluted with

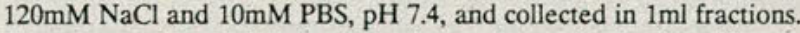
Fluorescence from labeled polymyxin was determined using a Turner model 112 fluorometer. Carbohydrate content in LPS was measured using an ACTA MVI spectrophotometer after fractions were oxidized with sodium periodate and condensed by Schiff's reagent. The results of my data shows that in the presence of LPS, fluorescein labeled polymyxin is recovered in the LPS fractions. Furthermore, when various concentrations of unlabeled polymyxin is added to the LPS and fluorescein labeled samples, a decreased amount of fluorescence is detected in the LPS fractions.

What has been concluded from this study is that fluorescein labeled polymyxin binds to several LPSs and this binding is inhibited by unlabeled polymyxin. This study indicates that polymyxin may be a suitable peptide for the development of an assay to detect endotoxins. 
CADMIUM DISRUPTS Ca2+-DEPENDENT CELL-CELL JUNCTIONS AND ALTERS THE PATTERN OF E-CADHERIN IMMUNOFLUORESCENCE IN LLC-PK CELLS. W.C. Prozialeck. Ph.D. R.J. Niewenhuis, Ph.D. and E.L. Fuentes, B.S... Philadelphia College of Osteopathic Medicine, Philadelphia, PA 19131.

Many of the toxic effects of $\mathrm{Cd}^{2+}$ in vivo appear to involve the disruption of junctions between cells in various endothelial and epithelial surfaces. Recent studies from our laboratories have shown that $\mathrm{Cd}^{2+}$ has fairly specific damaging effects on the adhering and occluding junctions between cells of the established porcine renal epithelial line, LLCPK1. The present studies were undertaken in order to further characterize the junction-perturbing effects of $\mathrm{Cd}^{2+}$ in LLCPK1 cells and to begin to identify the mechanisms underlying these effects.

LLC-PK 1 cells were grown to confluency on Falcon Cell Culture Inserts and exposed to $\mathrm{Cd}_{2}+$ by adding $\mathrm{CdCl}_{2}$ to either the apical or the basolateral compartments. The integrity of cell-cell junctions was assessed by monitoring the transepithelial electrical resistance. The results showed that exposure to $\mathrm{Cd}^{2+}(10-20 \mathrm{mi} \mathrm{croM})$ for $1-4$ hours caused a pronounced decrease in transepithelial resistance without killing the cells or causing them to detach from the growing surface. This decrease in resistance occurred more quickly and was more pronounced when $\mathrm{Cd}^{2+}$ was added to the basolateral compartment than when it was added to the apical compartment. These effects of $\mathrm{Cd}^{2+}$ were enhanced when $\mathrm{Ca}^{2+}$ was present at low concentrations $(0.1 \mathrm{mM})$ and were greatly reduced when $\mathrm{Ca}^{2+}$ was present at high concentrations $(10 \mathrm{mM})$. Additional studies showed that $\mathrm{Cd}^{2+}$ decreased the ampunt of E-cadherin, a Ca2+-dependent cell-adhesion molecule, that was associated with cell-cell junctions. These results indicate that $\mathrm{Cd}^{2+}$ disrupts the junctions between LLC-PK 1 cells by interacting with E-cadherin or a similar Ca2tsensitive site that is oriented toward the basolateral cell surface. (Supported by NIH Grant ESO5656).

\section{0}

NOCICEPTIVE REFLEXES AND THE SOMATIC DYSFUNCTION: SUPPORTING DATA FROM ANIMAL MODELS OF SPINAL FIXATION. B.J.Winterson \& D.J.Mokler. Departments of Physiology and Pharmacology, Univer ity of New England, College of Osteopathic Medicine, Biddeford ME 04005

Recently a model of somatic dysfunction was proposed in which the restrictions in mobility and autonomic, visceral and immunologic changes are produced by pain-related sensory neurons and their reflexes (Van Buskirk, JAOA 90,1990$)$. In this model, the ability of nociceptors to elicit guarding reactions is invoked as a possible cause of the abnormal musculoskeletal positions and restricted range of motion manifest in somatic dysfunction. The purpose of this presentation is to revise and extend this model in light of recent evidence from studies of the flexion response in the rat.

In the rat, injurious stimulation results in hyperexcitability of the flexion reflex and/or persistent flexion. These changes in the flexion reflex are known to last for hours, days and weeks. The hyperexcitability appears to be induced by stimulation to $\mathrm{C}$-fibers, and involve the activation of a particular type of excitatory amino acid receptor, the N-methyl-D-aspartate (NMDA) receptor. The NMDA receptor has been implicated elsewhere in the CNS in neuronal hyperexcitability, e.g. epilepsy and circuits for learning and memory. The idea of a spinal memery for injury is added to the model and a revision of the notion of the vicious cycle is discussed. Also, the flexion reflex is modulated by 5 -hydroxytryptaminergic and catecholaminergic agents. The revised model postulates possible peripheral and central sites of action for these agents. The therapeutic implications of the revised and extended model of somatic dysfunction will be discussed. (Supported by AOA grant 90-14-290)
OSTEOPATHIC MANIPULATIVE TREATMENT AND ELECTROMYOGRAPHY C. Steiner D.Q. H.K.Park M.D.and N.Guzelsu Ph.D.

UMDNJ-SOM Biomechanics and Rutgers University Biomedical Engineering, Piscataway,NJ,08854

In this study, we used electromyography(EMG) to analyze the effects of osteopathic manipulation on patients with low back pain due to muscle spasm. Patients with low back pain and controls who have never experienced back pain between the ages of 18 to 65 years were used in this study. Surface electrodes were adopted to collect the EMG data as a non-invasive method. Patients performed predetermined isometric tasks resisting flexion for varied weight levels $(0$ to $5 \mathrm{Kg})$ in a standing position before and after osteopathic manipulative treatment. The treatment was done 4 times within 2 weeks and patients were evaluated by the EMG measurements 7 times. The EMG recording of the back muscles was done 2048 times per second for 12 seconds by a 12 bit analog-digital converter in the computer. Volunteer control groups were tested 3 times, similar to the patients. Controls received manipulative treatment during a single session in order to evaluate the placebo effect of the treatment.

Integrated EMG data versus flexion load was investigated by linear regression for their slope values. The statistical analysis(ANOVA) of the controls total back muscles did not show a significant change in slope between the measurements However, the patients' slope value of total back muscles showed changes due to the manipulative treatment. The slope change in patients was further analyzed by using different high pass filtering techniques in order to find out the EMG values which produce maximum changes of back muscle activity (EMG versus external weights slopes) before and after treatment.

It is well established that EMG frequency decreases linearly during isometric contractions in time, due to muscle fatiguing. The high frequency components of the EMG signal decreased while the amplitudes of low frequency signal increased during the fatigue contraction. This is due to the changes in the firing rates of the motor unit and changes in the conduction velocity of the motor unit action potential. Power spectrum of EMG data is also analyzed for different types of patient (acute and chronic). The mean and median frequency of patients and controls' EMG was calculated by using Fast Fourier Transform. The changes in the frequency components of the surface EMG during the tasks before and after treatment reflects the change of muscle spasm and the involvement of the fatigue component in low back pain.

\section{2}

CHALLENGES OF RESEARCH WITH FRAIL PATIENTS IN AN OUTPATIENT GERIATRIC ASSESSMENT PROGRAM J.A. KNEBL DO, TCOM DIVISION OF GERIATRICS, FT WORTH, TX 76107 T.J. FAIRCHILD PHD, UNT CENTER FOR STUDIES IN AGING, DENTON, TX 76203

Recently there has been a growing interest in developing alternative health care models to meet the chronic needs of frail older patients. One alternative is a Gerontology Assessment and Planning (GAP) program. This model employs an interdisciplinary team to assess the frail persons physical as well as their psychosocial needs. The objective of this paper is to describe the challenges faced by clinicians and researchers attempting to do patient based research in a GAP.

The development and implementation of a research agenda has had to overcome three challenges. First, differences between clinical and research needs. Second, different professional orientation to research and clinical practice. Third, the inherent need of an assessment program to collect a wide range of physical and psychosocial data. Lessons learned from this experience suggest the need to keep a "patient first" framework in mind when planning research with frail patients in a GAP environment. This approach results in selecting research methods and instruments that have first clinical and then statistical significance. The implications of this approach will be discussed for improving interdisciplinary research with frail elderly in a clinical setting. 
FOURNIER'S GANGRENE: THE ROLE OF THE PRIMARY CARE PHYSICIAN

B. F. Schwartz, D.O.

Madigan Army Medical Center

Department of Surgery, Division of Urology

Tacoma, Washington 98431-5195

synergistic necrotizing fasciitis of the male genitalia, or Fournier's Gangrene, represents a true urologic emergency. Because up to two thirds of Fournier's patients have underlying Diabetes Meliitus and a significant percentage have a history of excessive alcohol intake, it is essential that the primary care physician be familiar with the recognition and prompt therapy of the disease. Failure to do so results in a very rapidly progressive and lethal process. In a review of the literature, various sources of infection have been implicated. These include: ischiorectal abscess, perirectal abscess, neurogenic bladder with ensuing urinary tract infection, indwelling Foley catheter, colorectal cancer, prostatic cancer and a number of others. The involvement of many organ systems further emphasizes the importance of the primary care physician to fully evaluate perineal and/or pelvic complaints in patients at risk for scrotal gangrene.

Presented here is a discussion of the subjective complaints, objective findings, diagnostic aids and treatment options in this intriguing but dangerous infection. In addition, we report a classic case of Fournier's Gangrene in a diabetic alcoholic who presented with perirectal pain and fever. The case reemphasizes the need for all primary care specialists to recognize the signs and symptoms of this rapidly fatal disease and know how to treat it.

\section{4}

MECHANISM OF DEFECTIVE POLY(ADP-RIBOSE) SYNTHESIS IN ISOLATED LYMPHOCYTES OF SLE PATIENTS. R.M Pertusi.D.O..H.Y.Chen M.D.M.S. B.R.Rubin D.Q.E.L. Jacobsen.Ph.D. Texas College of Osteopathic Medicine, University of North Texas, Ft. Worth.

Roly(ADP-ribose) polymerase is a nuclear enzyme which is activated by DNA strand breaks and is believed to function in the malntenance of chromatin structure during DNA repair. This enzyme utilizes $\mathrm{NAD}^{+}$as a substrate to form large complex polymers of ADP-ribose. Antibodies to polymers of ADP-ribose have been observed in patients with either idiopathic or druginduced SLE. Assays of poly(ADP-ribose) polymerase activity in lymphocytes from SLE patients showed a $70 \%$ decrease in activity (Sibley, et al. Arthritis and Rheumatism 32, 1045-1049, 1989). We have initiated studies to determine the mechanism of altered ADP-ribose polymer synthesis in lymphocytes from patients with SLE. Using an improved assay, we studied 15 patients fulfilling 4 or more ARA 1982 Revised Criteria for SLE and 13 age and sex matched controls. All controls tested, with one exception, had poly(ADP-ribose) polymerase activities greater than 140 units (Range $=140$ to 286). Sixty percent of the SLE patients demonstrated activities below the minimum activity observed in the control population (Range $=3.290$ ). The average activity for SLE patients $(111 \pm 80)$ was significantly lower than controls $(189 \pm 59)(p<0.0005)$. The relative $\mathrm{Km}$ of poly(ADP-ribose) polymerase as well as NAD stability (measured by HPLC) and glycohydrolase activity [which controls poly(ADP. ribose) degredation] did not differ between SLE and controls. These data suggest that decreased poly(ADP-ribose) polymerase activity in SLE may be due to a decreased number of active poly(ADP-ribose) polymerase molecules or altered polymerase activity.
THE NON-STRUCTURED ASSIGNMENT: INDEPENDENT STUDY AND ITS ROLE AS A STIMULUS FOR THE DEVELOPMENT OF A STUDENT PHYSICIAN'S OSTEOPATHIC PHILOSOPHY

\author{
Stacey L. Bushnell, B.S \\ Antheny G. Chila. D.O \\ Ohio University College of Osteopathic Medicine \\ Athens, Ohio
}

The predoctoral student of osteopathic medicine is generally exposed to a rigorous curriculum of basic sciences. The contemporary explosion of scientific knowledge in many of these disciplines renders the thoughtful assimilation of factual material nearly impossible. For the Phase I student, this is particularly noteworthy in the study of anatomy.

Andrew Taylor Still frequently emphasized that the osteopath's most important lessons were to be learned from the study of anatomy. This emphasis was used to support Still's view of diseases as effects occurring in regions of the body. A proper understanding of functional anatomy and physiologic compromise would make the use of manipulation basic to osteopathic medica practice. Still advocated this view in his argument for implementing a philosophical basis for the management of disease in general.

This report recounts the experience of a Phase II predoctoral student asked to undertake a project intended to address Still's considerations. A nonstructured cadaveric dissection assignment was utilized to stimulate the synthesis and integration of factual material presented during the student's Phase I program of study in anatomy. Dissection and observation about the anatomical relationships of the vagus nerve was the focus of the assignment.

The learning process encountered in completing this assignment demonstrated the student's ability for independent study. The fundamental idea of continuity between gross structure and cellular level functions was reinforced. Awareness of interdisciplinary integration was enhanced through search of literature. The significance of palpatory findings associated with somatic dysfunction was appreciated through participation in a concurrent clinical study.

Supported by the Norman J. Larson Clinical Research Fellowship, 1990 Funding was provided by the Norman J. Larson Clinical Research Fund.

\section{6}

RELATION BETWEEN FORCE OUTPUT AND EMG ACTIVITY FOLLOWING EXERCISE-INDUCED SKELETAL MUSCLE INJURY. J.N. Howell, Ph.D., G. Chleboun, M.S., C. Dawson, B.S., and R. Conatser, M.S. Ohio Univ. Coll. of Osteopathic Med., Athens, $\mathrm{OH} 45701$.

We examined the relation between force output and EMG activity in human muscle after exerciseinduced injury. Subjects repeatedly lowered a heavy load, normalized to their own strength, with their elbow flexors, until they could no longer control the rate of lowering. This typically took fewer than 10 repetitions and was repeated 3 times. Maximum isometric strength fell by more than $40 \%$ on the day after the exercise and returned only to $80 \%$ of control values 14 days later. The relation between force and EMG activity was linear over a range of submaximal contractions both before and after exercise, the slope expressing a ratio of force to EMG. This slope dropped by $69 \%$ immediately after exercise, but returned gradually to normal 4 days later, at a time when the maximum force capability of the muscle was still $30 \%$ below preexercise values. A similar pattern of force/EMG ratio was seen with maximum contraction, except that the ratio did not return fully to control values. Injured fibers, initially capable of generating action potentials, but little force, apparently become electrically silent within 4 days after the injury. (Supported by the Am. Osteopathic Assn. Grant \#90-08-259 and by the Ohio University College of Osteopathic Medicine.) 
ATYPICAL MONONUCLEOSIS PRESENTING AS TICK-BORNE INFECTION: A CASE STUDY

M. A. Ramirez, D.O., A.B.D. is an Intern at Doctors Hospital, Columbus, $\mathrm{OH}$

A. J. Silvagni, D.O., Pharm.D., is Assistant Dean for Special Projects at The University of Osteopathic Medicine and Health Sciences, Des

Moines, IA 50312

The current attention to Lyme Disease and other tick-borne infections can overshadow how these zoonoses mimic the signs, symptoms and laboratory results of viral and bacterial infections. The history of tick bite necessitates the careful evaluation of the patient for an extensive list of zoonotic diseases. The findings of fever, malaise, myalgia and headache lend further credibility to the diagnosis of tick-borne disease. The differential diagnosis of febrile tick-borne diseases includes Rocky Mountain Spotted Fever, Lyme Disease, Colorado Tick Fever, babesiosis, tularemia, relapsing fever, enterovirus, toxic shock syndrome, typhoid fever, scarlet fever, leptospirosis, viral hepatitis, cytomegalovirus and mononucleosis.

Mononucleosis may present with an unpredictable and uncharacteristic pattern of symptoms in patients over 30 years of age. The physician must be cautious not to neglect the possibility of atypical mononucleosis or other non tick-borne infections. In the older age group, the diagnosis is based upon the laboratory picture. In the case presented, the clinical course, negative serology for Lyme Disease, rickettsial disease and Colorado Tick Fever combined with the positive heterophile antibody and Monospot tests allowed for the definitive diagnosis of monnnucleosis. This case illustrates the need for vigilance to guard against the overdiagnosis of tick-borne diseases.

\section{8}

EIGHT MONTHS OF ENDURANCE EXERCIBE TRAINING (EET) ALTERS BARORECBPTOR RESPONBIVENESS (BRR) TO INFUBIONS OF PHENYLEPHRINE HCL (PE).

G.H.J. Stevens MS, B.H. Foresman DO, and P.B. Raven PhD. Texas college Osteopathic Medicine, Depts. Physiology \& Medicine, Fort Worth, Tx, 76107.

We have previously shown using cross-sectional data that EET individuals have a decreased BBR as defined by change in heart rate per change in mean arterial pressure ( $\triangle \mathrm{HR} / \triangle \mathrm{MAP}$ ) to $\mathrm{PE}$. In the present investigation we assessed this relationship following 8 months of EET. Eight males participated in the study with $\bar{x}$ age $27.6 \pm 4.03$ yrs. During the 8 months of EET maximal oxygen consumption increased from $45.02 \pm 6.12$ to 57.46 $+7.85 \mathrm{ml} / \mathrm{kg} / \mathrm{min}(\mathrm{p}<0.05)$. During $\mathrm{PE}$ infusions the subject had an 18 gauge catheter placed in an arm vein and rested supine during a 30 minute equilibration period. PE was infused during 5,6 minute stages using an infusion pump. The stages were $24,48,60,90$, and $120 \mu \mathrm{g} / \mathrm{min}$. During the 4 th minute of each stage HR, blood pressure, and forearm blood flow (FBF) were collected. EET resulted in higher resting FBFs and decreased forearm vascular resistances (FVR). The EET individuals, however, constricted their periphery to the same extent by $120 \mu \mathrm{g} / \mathrm{min}$ of $\mathrm{PE}$. In examining $\triangle H R$ from baseline (BL) vs $\triangle$ MAP from $B L$ the EET bradicardic response plateaued e $60 \mu \mathrm{g} / \mathrm{min}$ vs UT who continued to decrease HR with increasing MAP. Finally, BBR to PE infusions decreased with training from 0.884 to $0.446(p<0.05)$. We conclude that 8 months of EET results in alterations in peripheral vascular responses to steady- state infusions of PE and decreases BRR.
LOCKED SCAPULA: A REHABILITATION CHALLENGE

D.R. Weiss, D.O., H. Wittels, M.D., L. Jacobs, M.D., Ph.D., C. Schneck, M.D., Ph.D.

Temple University Hospital, Physical Medicine and Rehabilitation

Philadelphia, PA 19140

Scapulothoracic dislocation (locked scapula) with intrathoracic herniation of the inferior border is a rare occurrence described only twice previously, once secondary to trauma and the other status post multiple rib resections. With the increased prevalence of cardiothoracic surgery and with the availability of more aggressive rehabilitation programs, the prevention and treatment of locked scapula takes on increasing importance. An in-depth investigation of the literature, prompted by a recent incident of locked scapula on our rehabilitation unit after posterior lateral thoracotomy, revealed no recommended treatment precautions to obviate this postoperative complication. A videofluoroscopic examination of the scapular movement relative to the posterior ribs during elevation, protraction and rotation of the shoulder in normal subjects was performed to determine movements that might increase the risk of scapulothoracic herniation in postoperative thoracotomy patients. Based on this study, a postoperative rehabilitation exercise program with appropriate precautions was developed. A treatment protocol with excellent functional outcome was also instituted for our patient who had a nonreducible dislocation.

\section{0}

EPIDURAL ANALGESIA: A BETTER TECHNIQUE. MJ Kilbride. DO; AJ Senagore, MD; WP Mazier, MD. Ferguson Hospital, Anesthesia Department, Grand Rapids, MI 49502

The optimal method of postoperative analgesia has long been debated The three most common techniques are intramuscular injection. (IM), patient controlled analgesia (PCA), and epidural catheter injection (EPI). The purpose of this study was to prospectively compare these three techniques for analgesia following major colorectal surgery. All patients undergoing major abdominal colorectal procedure were eligible for study. Patients were randomized to one of three analgesic groups: Group I $(\mathrm{N}=19)$ received intramuscular morphine sulphate; Group II $(\mathrm{N}=22)$ patients utilized patient controlled morphine sulphate; Group III $(\mathrm{N}=23)$ received epidural morphine sulphate. Data collected was age, time to first bowel movement (Time BM), amount of narcotic, number achieving $75 \%$ of preoperative forced vital capacity within 7 days (FVC 75\%). Using an analog pain score patients were assessed for incidence of excellent pain relief (score $\geq 2.5$ ). Data (mean \pm SL)

$\begin{array}{cccll}\text { GRP } & \text { Age } & \text { Amt Narc } & \text { FVC75\% } & \text { Time BM } \\ \text { I } & 64.6 \pm 2.9 & 215 \pm 24 & 8 / 19 & 5.5 \pm 0.7 \\ \text { II } & 57.8 \pm 3.1 & 355 \pm 31 & 17 / 21^{*} & 5.1 \pm 0.3 \\ \text { III } & 60.5 \pm 3.5 & 81 \pm 6^{*} & 16 / 23^{*} & 5.0 \pm .3 \\ & \text { NUMBER WITH EXCELLENT PAIN RELIEF } & \\ \text { GRP } & \text { Day 1 } & \text { Day 2 } & \text { Day 3 } & \\ \text { I } & 1 / 19 & 7 / 19 & 2 / 9 & \\ \text { II } & 8 / 22 & 8 / 22 & 9 / 20 & \\ \text { III } & 14 / 23 \# & 14 / 23 \# & 14 / 22 \# & \end{array}$

*Student's t-test; \#Fisher's exact; $\mathrm{p}<.05$

Conclusions: The data indicate that epidural catheter injection achieves excellent pain control in a greater percentage of patients while utilizing a much lower dose of narcotics. Both PCA and EPI allow faster return to preoperative ventilatory status compared to IM. 